\title{
Hyperbaric Oxygen Therapy Improves Cognition in Patients Severe TBI; A Prospective Study
}

\section{Sankaran $\mathrm{R}^{*}$ and Reshma KK}

*Corresponding author: Sankaran R, Department of Physical Medicine and Rehabilitation, Amrita Institute of Medical Sciences, Cochin, Kerala, India, 682041, Tel: 285-484-1234, E-mail: ravisankaran@aims.amrita.edu

Citation: Sankaran R, Reshma KK (2020) Hyperbaric Oxygen Therapy Improves Cognition in Patients Severe TBI; A Prospective Study. J Neurol Neurol Disord 6(1): 102. doi: 10.15744/2454-4981.6.102

Received Date: July 15, 2020 Accepted Date: December 28, 2020 Published Date: December 30, 2020

\begin{abstract}
Background: Cognitive impairment post TBI is an important factor in rehabilitation outcomes. Pharmacological and therapeutic services do not directly address damage to the cortical infrastructure. Hyperbaric Oxygen Therapy is known to improve brain function and healing.

Objective: Report outcomes of Hyperbaric Oxygen Therapy in people with Severe TBI

Materials and Methods: The study was conducted in a tertiary level rehabilitation centre as a Prospective, non-randomized, case control study. Thirty-seven people with severe TBI (18 cases, 19 controls) participated. At baseline the following were considered: age and gender, level of education, duration of acute care length of stay, duration of post-traumatic amnesia, associated complications, or comorbidities. The main outcome measures were Disability Rating Score, Montreal Cognitive Assessment. DRS was taken at the beginning of Acute Inpatient rehabilitation and at the end along with before and after the intervention period. MoCA was taken at the beginning and end of the intervention period. No one suffered adverse effects of the treatment.
\end{abstract}

Results: Subjects in both groups were similar at baseline. After the intervention there was a significant change in MoCA favoring the HBOT group. There was no difference in DRS at any interval.

Conclusion: HBOT can improve cognitive performance after Severe TBI in the post-acute phase. This can improve long term quality of life and facilitate return to society.

Keywords: Traumatic Brain Injury; Hyperbaric Oxygen Therapy; MoCA; DRS; Cognition

List of abbreviations: TBI: Traumatic Brain Injury; QoL: Quality of Life; ADL: Activities of Daily Living; MoCA: Montreal Cognitive Assessment; DRS: Disability Rating Scale; HBOT: Hyperbaric Oxygen Therapy; AIR: Acute Inpatient Rehabilitation

\section{Introduction}

Traumatic brain injury (TBI) is a significant cause of morbidity. In the USA the average lifetime cost of care for a severe TBI patient ranges from $\$ 600,000$ to $\$ 1,875,000$ [1]. In India 1.6 million persons sustain TBI annually. Of those 200,000 will die [2]. The survivors face a spectrum of challenges most commonly related to cognitive or corticospinal tract dysfunction. Brain injury related neuropsychological impairment affects quality of life (QoL) [3]. The common presentations of this are: impaired concentration, decreased attention, easy distractibility, impaired visuo-spatial conceptualization, slow verbal/ visual stimulus processing, impaired memory, communication disorder, poor judgment, poor executive function [4]. Participation is a strong predictor of life satisfaction in the differently-abled. These issues lead to ADL dependence when they result in depersonalization [5]. The standard treatment consists of pharmacological agents and therapeutic exercises. Pharmacological agents used vary from agents like Citocholine to Amantadine [6]. Psychological intervention is: Attention process training and tasks for attention deficits, compensatory strategies and errorless learning training for memory deficits, pragmatic language skills and social behavior guidance for cognitive-communication disorder, meta-cognitive strategy, and problem-solving training for executive disorder are the mainstay of therapy for cognitive deficits in persons with TBI [7]. None of these directly address the cortical infrastructure damage. Often, they work with what is preserved.

Hyperbaric Oxygen therapy (HBOT) has been shown to reduce intracranial edema, promote angiogenesis, improve overall brain function, and lead to less complications and better outcomes when given in the acute phase of treatment [8]. It is also known to improve QoL and functional outcomes [9] up to three years post event. The existing studies don't prospectively look at changes in specific domains of cognition after severe TBI. 
We did this study to see if HBOT in the subacute phase could specifically improve cognition and functional levels. Our objectives are: 1) report the difference in Montreal Cognition Assessment (MoCA), and 2) report the differences in Disability Rating Scale (DRS) between the two groups.

\section{Methods}

The design is a non-randomized prospective study developed in the first author's institute. Ethics board approval was obtained (IEC- PMR-165). Patients were taken from the Acute Inpatient Rehabilitation service setting between 2017 and 2019 in a tertiary level hospital. Thirty-seven patients participated after taking consent. Patients were screened for duration from injury. Inclusion criteria were: Severe Traumatic Brain Injury admitted in the Acute Inpatient Rehabilitation service, Exclusion criteria were: Depression or other psychiatric conditions, Duration of Acute inpatient care $>4$ months, Post-traumatic amnesia $>4$ months, Concomitant neurological conditions, recovering from LRTI, with albumin less than 3.0., MRI with no focal lesion or evidence of Diffuse Axonal Injury. Each patient received 10 sessions of 100\% Oxygen at 2 ATA for one hour.

At baseline the following were considered: age and gender, level of education, duration of acute care length of stay, duration of post-traumatic amnesia (measured with the Abbreviated Westmead Post Traumatic Amnesia Scale), associated complications, or comorbidities. The outcome measures used were DRS and MoCA. The DRS was taken at induction to AIR (Acute Inpatient Rehabilitation) at discharge from AIR, and again before and after HBOT for the cases. In the controls this was repeated at onemonth follow-up and again at the second month follow-up. DRS is validated for use in acquired brain injury [10]. MoCA was used pre and post intervention for cases whereas at one-month follow-up and again at the second month follow-up for the controls. While MOCA was designed as a screening tool for cognitive impairment [11], it has been validated for serial use if at least a one-month interval was given between retests [12,13]. The total possible score is 30 points with a score of 26 or more considered normal. It assesses the domains of attention and concentration, executive function, memory, language, visuo-constructional skills, conceptual thinking, calculation, and orientation. To better adjust the MoCA for lower educated individuals, add 2 points to the total MoCA score for those with 4-9 years of education and 1 point for 10-12 years of education [14]. Assessment was done by the other author. Selection was by patient/ party choice, after being informed of possible risks and benefits. HBOT $100 \%$ O 2 at 2 bar pressure for 1 hour combined with compression and decompression for a total of 2 hours. Each patient received 20 sessions. Prophylactic myringotomy was not done. Concomitant Physical and Occupational therapy was done whether or not they received the intervention. As all these patients were ambulating with various degrees of support so the goals of therapy were to maximize the available function. Oral antispastics were not utilized as they could confound outcomes. For statistical analysis we used mean and standard deviation to define the dispersion. Baseline data were assessed using Pearson's chi-square test. Levene's test for equality of variance and two-tailed t-test were used for ordinal outcome measures.

\section{Results}

Thirty-seven patients who satisfied the inclusion criteria were recruited during the study period. The mean age of the participants was $42.86 \pm 8.71$ years. All participants met inclusion criteria. All of them were on an anti-epileptic agent before and throughout the duration of treatment. Among participants, 18 (48\%) patients chose HBOT, 19 (52\%) remained as controls.

There was no significant difference in the baseline variables between cases and controls for age, gender, level of education, being currently depressed, duration of acute care length of stay, duration of post-traumatic amnesia (measured with the Abbreviated Westmead Post Traumatic Amnesia Scale) score, associated complications, comorbidities, education.

On average the HBOT recipients were worse for acute Los, PTA duration, younger, had more complications, and more patients with only a $12^{\text {th }}$ standard education than the control group (Table 1 ).

\begin{tabular}{|c|c|c|}
\hline Gender & Case & Control \\
\hline Age average & M 15: F 3 & M 18: F 1 \\
\hline Level of education & 44.52 & 41.22 \\
\hline Acute Length of stay & $\begin{array}{c}12^{\text {th }}-3 \\
\text { Diploma- 5 } \\
\text { Degree- 10 }\end{array}$ & $\begin{array}{c}12^{\text {th }} \text { - } 1 \\
\text { Diploma- 11 } \\
\text { Degree- } 8\end{array}$ \\
\hline Comorbidities & $1.95 \pm 0.81$ & $1.5 \pm 0.49$ \\
\hline Duration of Post Traumatic Amnesia & $1.63 \pm 0.27$ & $1.9 \pm 0.72$ \\
\hline Complications & HTN 4/ DMII 3 & HTN 5/ DMII 5 \\
\hline & $\begin{array}{c}\text { Spasticity- 5 } \\
\text { Hemicraniectomy- 5 } \\
\text { Tracheostomy- 7 } \\
\text { Polytrauma- 3 } \\
\text { Critical Illness Neu- } \\
\text { ropathy- 1 } \\
\text { Hemineglect- 1 }\end{array}$ & $\begin{array}{c}\text { Spasticity- } 4 \\
\text { Tracheostomy- } \\
\text { Polytrauma- 2 } \\
\text { Brachial plexus } \\
\text { injury- 1 } \\
\text { Cerebellar ataxia-1 }\end{array}$ \\
\hline
\end{tabular}

HTN- Hypertension, DMII- Diabetes Mellitus Type 2

Table 1: Baseline details 
We compared outcomes between cases and controls. Based on the results, patients had a significant improvement in MoCA after HBOT (Table 2). There was no significant difference between the groups at any interval for DRS, nor for baseline MoCA. There was a significant difference between the groups for MoCA post- HBOT favoring the cases (Figure 1).

\begin{tabular}{|c|c|c|c|}
\hline & Case & Control & p-value \\
\hline Pre- AIR DRS & $27.55 \pm 4.1$ & $27.5 \pm 3.8$ & 0.87 \\
\hline Post- AIR DRS & $8.2 \pm 4.9$ & $7.88 \pm 7.3$ & 0.79 \\
\hline Pre-intervention DRS & $3.5 \pm 6.1$ & $3.33 \pm 4.8$ & 0.95 \\
\hline Post-intervention DRS & $2.25 \pm 4.5$ & $2.63 \pm 3.6$ & 0.91 \\
\hline Pre-intervention MoCA & $8.45 \pm 3.61$ & $8.88 \pm 2.12$ & 0.81 \\
\hline Post-intervention MoCA & $20.15 \pm 5.18$ & $13.4 \pm 4.31$ & 0.001 \\
\hline
\end{tabular}

AIR- Acute Inpatient Rehabilitation

DRS- Disability Rating Score

MoCA- Montreal Cognitive Assessment

Table 2: Outcomes

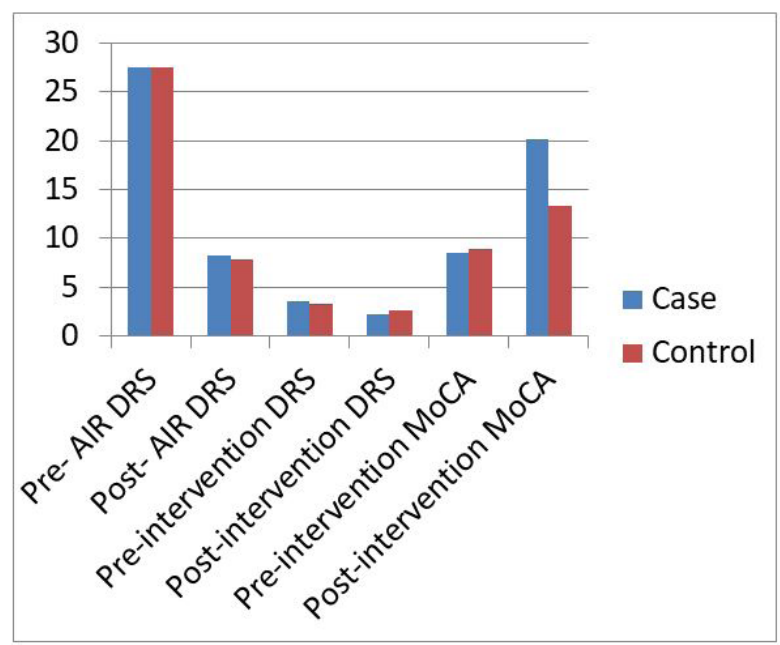

Figure 1: Outcomes

\section{Discussion}

Based on the results, HBOT given to patients with Severe TBI resulted in better cognitive function in the subacute phase. From baseline to follow-up and pre-intervention they were not significantly different. This is because once the patient is medically stable, they are transferred to AIR. Once they can walk with assistance they are planned for discharge. The spectrum of therapy services until the first follow-up is often limited to just Physical Therapy. We looked at overall functional capacity, length of stay, duration of PTA, education, co-morbidities, and associated complications. All of the patients were on Amantadine in the AIR phase, and on an antiepileptic throughout the study timeframe. There was no significant difference in the DRS despite the cognitive improvement. This is possibly due to a short follow-up frame, and some of our patients being retired already. The domains in MoCA that improved were attention, memory, visuospatial, delayed recall and orientation. This has not been reported in prior studies.

Recent studies on the role of HBOT in recovery from various types of brain injury have reported improvement and recovery $[8,15]$. These are with respect to ADLs and recovery of consciousness. This study is different in that it prospectively looks at cognitive recovery as the primary outcome. The only other one similar is in HIE patients and is a retrospective review [9].

The goal of rehabilitation is to make a person as independent as possible considering their primary disease and co-morbidities. TBI related neuropsychological impairment affects quality of life (QOL) [16]. Cognitive deficits post-TBI are rarely domain specific [17]. Patients often report, difficulty interpreting others speech and intentions [18]. Three- fourths have memory and attention disorders (75\%) [19]. As decision making demands increase, memory impairment manifests as time- and event-based difficulty [20]. These are mediated by executive networks.

Two main networks govern executive function. Cold processes (control of attention and cognition) localize to the dorsolateral network. Hot processes localize to the orbito-frontal/ ventro-medial pathways. TBI disrupts both networks. Hot deficits can compromise relationships, functional independence and mental capacity in daily life [21]. Perception, learning, and memory are dependent on synchronization of neural activity across brain regions. Suboptimal synchronization manifests as impaired cognition. Neuronal degeneration post-TBI, alters inter-circuit communication. While highly synchronized circuits are less susceptible to damage, subtle frequency changes can be problematic for daily life [22]. Synchronization of neural activity across brain regions is critical to processes that include perception, learning, and memory. 
Neuron loss, diffuse axonal injury, microbleed, and blood-brain barrier (BBB) disruption contribute to the development of cognitive impairment [23]. Cerebrovascular alterations occur early in TBI, leading to brain edema. One mechanism is by complement activation [24]. Significant elevations C4b, factor D, Bb, MBL, C3b and C5b-9 terminal C complex, and depressions of CR1 and CD59 are associated with acute TBI [25]. Another mechanism is by excessive activation of N-methyl-D-aspartate receptors (NMDARs). This leads to increased Inducible nitric oxide synthase (iNOS). This mediates inflammation and exacerbates damage in TBI. Its expression increases with time. Inhibiting iNOS results in better synaptic plasticity. This improves Long Term Potentiation, hence synaptic activity [26]. HBOT acts on both mechanisms. It reduces complement activation [27] and decreases inducible Nitric Oxide Synthase (iNOS) activity/expression. It does so by decreasing phosphorylation of ERK1/2 and Akt resulting in decreased activity of NFKB [28]. This is efficacious in Acquired Brain Injury. Meta-analysis shows faster recovery of consciousness in TBI patients with shorter length of stay and complications [29]. A recent publication in acute ischemic stroke shows recipients had less morbidity, mortality, and faster resolution of intracranial edema [8]. Another study using this in Hypoxic Ischemic encephalopathy revealed statistically significant differences in return to consciousness compared to controls who received this in the first 3 months post injury [15].

Who needs this treatment modality? The optimal patient has an average innate potential to improve. Comorbidities and MRI findings with predominantly focal lesions and less Diffuse Axonal Injury. The following factors associate with greater cognitive dysfunction: lower educational level, [30] length of Loss of Consciousness [31], duration of post traumatic amnesia, duration of hospital stay and volume of diffuse axonal injury [32]. Naturally a patient without such factors has less to improve on. While these predict neuro-cognitive outcomes, they present an incomplete picture. What happens after acute care discharge, and inability to predict quality of rehabilitation services are missed. In a 10-year longitudinal study by Sigurdardottir et al, 94-96\% stabilized in function after the first year [33]. This leaves a narrow window within which to maximize a patient's potential gains.

Knowing all this, when is the best time to implement this modality? Earlier is better. When the goal is to improve cognition, the patient should be able to complete a test. These assume speech, comprehension, repetition, and motor skills are sufficiently preserved. The current spectrum of treatment options either seek to pharmacologically reduce inflammation or supplement depleted neuro-hormones based on clinical presentation. While all of these are fundamental to care, they don't directly restore brain connectivity. This is critical to subsequent therapy services. The limitations of this study are as follows: Non-randomized design, selection bias, use of a detailed cognitive test would yield better definition of what functions improve with this treatment.

\section{Conclusion}

Hyperbaric Oxygen therapy given in the subacute phase after severe traumatic brain injury can result in improved cognitive function.

\section{References}

1. Rehabilitation of persons with traumatic brain injury (1998) NIH Consensus Statement 16: 1-41.

2. Gururaj G (2002) Epidemiology of traumatic brain injuries: Indian scenario. Neurol Res 24: 24-8.

3. Pettemeridou E, Kennedy MRT, Constantinidou F (2020) Executive functions, self-awareness and quality of life in chronic moderate-to-severe TBI. Neurorehabilation 46: 109-18.

4. Barman A (2016) Cognitive Impairment and Rehabilitation Strategies After Traumatic Brain Injury. Indian J Psychol Med 38: $172-81$.

5. Pierce C, Hanks RA (2006) Life satisfaction after traumatic brain injury and the World Health Organization model of disability. Am J of Phys Med Rehab 85: 889-98.

6. Talsky A, Pacione LR, Shaw T, Wasserman L, LennyAM, et al. (2011) Pharmacological interventions for traumatic brain injury. BCMJ 53: 26-31.

7. Barman A, Chatterjee A, Bhide R (2016) Cognitive Impairment and Rehabilitation Strategies After Traumatic Brain Injury. Indian J Psychol Med 38: 172-81.

8. Sankaran R, Ramachandran SN (2019) Hyperbaric Oxygen therapy in Acute Ischemic Stroke. Indian J Appl Res 9: 10.36106/ijar.

9. Hadannya A, Golan H, Fishlev G, Bechor Y, Volkov O (2015) Hyperbaric oxygen can induce neuroplasticity and improve cognitive functions of patients suffering from anoxic brain damage. Restor Neurol Neurosci 33: 471-86.

10. Gouvier WD (1987) Reliability and validity of the Disability Rating Scale and the Levels of Cognitive Functioning Scale in monitoring recovery from severe head injury. Arch Phys Med Rehabil 68: 94-7.

11. Nasreddine ZS, Phillips NA, Bédirian V, Charbonneau S, Whitehead V, et al. (2005) The Montreal Cognitive Assessment, MoCA: a brief screening tool for mild cognitive impairment. J Am Geriatr Soc 53: 695-9.

12. Costa AS, Fimm B, Friesen P, Soundjock H, Rottschy C, et al. (2012) Alternate-form reliability of the Montreal cognitive assessment screening test in a clinical setting. Dement Geriatr Cogn Disord 33: 379-84.

13. Nasreddine ZS, Rossetti H, Phillips N, Chertkow H, Lacritz L (2012) Normative data for the Montreal Cognitive Assessment (MoCA) in a population-based sample. Neurol 78: 765-6.

14. Malek-Ahmadi M, Powell JJ, Belden CM, O'Connor K, Evans L, et al. (2015) Age- and education-adjusted normative data for the Montreal Cognitive Assessment (MoCA) in older adults age 70-99. Neuropsychol Dev Cogn B Aging Neuropsychol Cogn 22: 755-61.

15. Sankaran R, Radhakrishnan K, Sundaram KR (2019) Hyperbaric oxygen therapy in patients with hypoxic ischemic encephalopathy. Neurol India 67: 728-31. 16. Pettemeridou E, Kennedy MRT, Constantinidou F (2020) Executive functions, self-awareness and quality of life in chronic moderate-to-severe TBI. NeuroRehabilitation 46: 109-18. 
17. Hurst FG, Ownsworth T, Beadle E, Shum DHK, Fleming J (2020) Domain-specific deficits in self-awareness and relationship to psychosocial outcomes after severe traumatic brain injury. Disabil Rehabil 42: 651-9.

18. Theadom A, McDonald S, Starkey N, Barker-Collo S, Jones KM, et al. (2019) Social cognition four years after mild-TBI: An age-matched prospective longitudinal cohort study. Neuropsychology 33: 560-7.

19. Nemkova SA (2019) Current possibilities of complex diagnosis and treatment of the consequences of traumatic brain injury, Zh Nevrol Psikhiatr Im S S Korsakova 119: 94-102.

20. Kumar RG, Gao S, Juengst SB, Wagner AK, Fabio A (2018) The effects of post-traumatic depression on cognition, pain, fatigue, and headache after moderateto-severe traumatic brain injury: a thematic review. Brain Inj 32: 383-94.

21. Wood RL, Worthington A (2017) Neurobehavioral Abnormalities Associated with Executive Dysfunction after Traumatic Brain Injury. Front Behav Neurosci 11: 195 .

22. Schumm SN, Gabrieli D, Meaney DF (2020) Neuronal Degeneration Impairs Rhythms Between Connected Microcircuits. Front Comput Neurosci 14: 18.

23. Wang ML, Li W (2016) Cognitive impairment after traumatic brain injury: The role of MRI and possible pathological basis. J Neurol Sci $370: 244-50$.

24. Dinet V, Petry KG, Badaut J (2019) Brain-Immune Interactions and Neuroinflammation After Traumatic Brain Injury. Front Neurosci 2019: 10.3389/ fnins.2019.01178.

25. Goetzl EJ, Yaffe K, Peltz CB, Ledreux A, Gorgens K, et al. (2020) Traumatic brain injury increases plasma astrocyte-derived exosome levels of neurotoxic complement proteins. FASEB 34: 3359-66.

26. Wang B, Han S (2018) Inhibition of Inducible Nitric Oxide Synthase Attenuates Deficits in Synaptic Plasticity and Brain Functions Following Traumatic Brain Injury. Cerebellum 17: 477-84.

27. Romuald O, Konarski M, Siermontowsk P (2017) Hyperbaric Oxygen Therapy (HBOT) as a Therapeutic Option for Patients with Atopic Dermatitis (AD). Polish Hyperbaric Research 60: 10.1515/phr-2017-0012.

28. Resanovic I, Gluvic Z, Zaric B, Sudar-Milovanovic E, Jovanovic A, et al. (2019) Early Effects of Hyperbaric Oxygen on Inducible Nitric Oxide Synthase Activity/ Expression in Lymphocytes of Type 1 Diabetes Patients: A Prospective Pilot Study. Int J Endocrinol 2019: 10.1155/2019/2328505.

29. Bennett MH, Trytko B, Jonker B (2012) Hyperbaric oxygen therapy for the adjunctive treatment of traumatic brain injury. Cochrane Database Syst Rev 12: 10.1002/14651858.CD004609.pub2.

30. Mollayeva T, Mollayeva S, Pacheco N, D’Souza A, Colantonio A (2019) The course and prognostic factors of cognitive outcomes after traumatic brain injury: A systematic review and meta-analysis. Neurosci Biobehav Rev 99: 198-250.

31. Bedard M, Taler V, Steffener J (2018) Long-term prospective memory impairment following mild traumatic brain injury with loss of consciousness: findings from the Canadian Longitudinal Study on Aging. Clin Neuropsychol 32: 1002-18.

32. Aldossary NM, Kotb MA, Kamal AM (2019) Predictive value of early MRI findings on neurocognitive and psychiatric outcomes in patients with severe traumatic brain injury. J Affect Disord 243: 1-7.

33. Sigurdardottir S, Andelic N, Røe C, Schanke A (2020) Trajectory of 10-Year Neurocognitive Functioning After Moderate-Severe Traumatic Brain Injury: Early Associations and Clinical Application. J Int Neuropsychol Soc 26: 1-14. 